\title{
FRANK SCHOLZE
}

Innovationspotential von Zusammenlegungen und Fusionen von Wissenschaftseinrichtungen am Beispiel des Karlsruher Instituts für Technologie (KIT) - Impulse für das Bibliothekssystem

Abstract

Das Karlsruher Institut für Technologie (KIT) ist am 01.10.2009 aus dem Zusammenschluss des Forschungszentrums Karlsruhe in der HelmholtzGemeinschaft und der Universität Karlsruhe hervorgegangen. Während sich an der Universität ein zweischichtiges Bibliothekssystem entwickelt hatte, das sich im Wandel zur funktionalen Einschichtigkeit befand, war am Forschungszentrum Karlsruhe von Anfang an ein einschichtiges Bibliothekssystem nach dem Vorbild einer Werksbibliothek eingeführt worden. Die Fusion zum KIT wirkte als Katalysator für ein pragmatisches Modell der Einschichtigkeit, bei dem Servicequalität und Effizienz der Informationsversorgung im Vordergrund stehen.

Keywords

Universität Karlsruhe, Forschungszentrum Karlsruhe, Helmholtzgemeinschaft, Fusion, Hochschulbibliothekssystem, Funktionale Einschichtigkeit

\section{Kurzbiographie}

Frank Scholze ist seit Januar 2010 Direktor der Bibliothek des Karlsruher Instituts für Technologie (KIT). Bevor er die Leitung der KIT-Bibliothek übernahm, war er als Referent im Ministerium für Wissenschaft, Forschung und Kunst Baden-Württemberg mit dem Aufbau elektronischer 
Informationsinfrastrukturen für Forschung und Lehre betraut und mehrere Jahre Leiter der Benutzungsabteilung der Universitätsbibliothek Stuttgart. Frank Scholze studierte Bibliothekswesen an der Hochschule der Medien Stuttgart, sowie Kunstgeschichte und Anglistik an der Universität Stuttgart.

Frank Scholze ist Mitglied einer Reihe von wissenschaftlichen Ausschüssen und Beiräten; unter anderem der Deutschen Forschungsgemeinschaft (DFG), des Fachinformationszentrums Karlsruhe, von DARIAH-DE (Digital Research Infrastructures for the Arts and Humanities) und der Deutschen Gesellschaft für Klassifikation (GfKI).

Das Karlsruher Institut für Technologie (KIT) und seine Vorgängereinrichtungen

Das Karlsruher Institut für Technologie (KIT) ist am 01.10.2009 aus dem Zusammenschluss des Forschungszentrums Karlsruhe in der HelmholtzGemeinschaft und der Universität Karlsruhe hervorgegangen. Die Helmholtz-Gemeinschaft ist die größte Wissenschaftsorganisation Deutschlands. In ihren 17 naturwissenschaftlich-technischen und biologisch-medizinischen Forschungszentren arbeiten insgesamt 31.000 Beschäftigte. Das jährliche Budget der Gemeinschaft beträgt mehr als 3,3 Mrd. Euro. Die Universität Karlsruhe war eine forschungsstarke Universität mit naturwissenschaftlich-technischem Schwerpunkt. Mit dem Zusammenschluss entstand eine der weltweit größten Forschungs- und Lehreinrichtungen mit knapp 9.000 Beschäftigten und einem jährlichen Budget von 730 Mio. Euro, mit dem Potenzial und dem Anspruch, in Gebieten wie der Energieforschung oder der Nanotechnologie eine Spitzenposition einzunehmen. Universität und Forschungszentrum hatten sich mit diesem Konzept bereits in der ersten Runde der Exzellenzinitiative, einem zur Förderung von Wissenschaft und Forschung an deutschen Hochschulen ausgelobten Förderprogramm, durchgesetzt. Das KIT war im Oktober 2006 eines von nur drei Zukunftskonzepten, die von Wissenschaftsrat und Deutscher Forschungsgemeinschaft (DFG) gefördert wurden. 
Damit begann ein einzigartiges Experiment in der Wissenschaftsgeschichte der Bundesrepublik Deutschland. Denn die Universität gehört dem Land Baden-Württemberg und das Forschungszentrum dem Bund. Und für beide gilt eigentlich ein striktes, im Grundgesetz verankertes Kooperationsverbot. Daher bedurfte es auch eines eigenen Gesetzes, um die Zusammenführung beider Einrichtungen rechtlich abzusichern. Hat auch die Euphorie durch das Ausscheiden in der zweiten Runde der Exzellenzinitiative einen Dämpfer erhalten, so richtet sich nach wie vor beträchtliche nationale und internationale Aufmerksamkeit auf die Entwicklung am KIT, da sie weiterhin einer der vielversprechendsten Ansätze für Spitzenforschung im technischnaturwissenschaftlichen Bereich ist.

\section{Bibliothekssystem der Universität Karlsruhe}

Die Universität Karlsruhe wurde 1825 als die erste Polytechnische Schule in Deutschland gegründet. Als Vorbild diente die École Polytechnique in Paris. Eine zentrale Bibliothek entstand erst 1840. Neben ihr bildeten sich zahlreiche in Verwaltung und Literaturauswahl eigenständige Institutsund Lehrstuhlbibliotheken mit teilweise recht großen und gut sortierten Beständen. Diese Bestände wurden nur intern erschlossen und standen in der Regel ausschließlich den Mitarbeitern der jeweiligen Einrichtung zur Verfügung. ${ }^{1}$

Ende der 1960er Jahre wurde nach der Errichtung eines zentralen Bibliotheksgebäudes an der Universität Karlsruhe damit begonnen, schrittweise ein Bibliothekssystem aufzubauen, wobei man sich an den Empfehlungen des Wissenschaftsrates und der Deutschen Forschungsgemeinschaft zur Zusammenarbeit zwischen den

1 Eckl, Liselotte u. Michael Mönnich: Das Bibliothekssystem der Universität Karlsruhe. In: EUCOR-Bibliotheksinformationen 3 (1993). S. 44-50. http://www.ub.unifreiburg.de/fileadmin/ub/eucor_infos/3-1993/09.html (26.10.2013). 
Hochschulbibliotheken und den Institutsbibliotheken orientierte. ${ }^{2}$

Beim Aufbau des Bibliothekssystems mussten aufgrund der Ressourcenknappheit Schwerpunkte gesetzt werden, die für die bibliothekarische Zusammenarbeit in der Universität und auch im Lande besonders wichtig waren und den größten Nutzeffekt versprachen. Diese waren insbesondere die Einrichtung eines Zeitschriftengesamtkataloges der Universität, Beratung und Hilfestellung für Institutsbibliotheken sowie die Schaffung größerer Bibliothekseinheiten. Hier sind die Fakultätsbibliotheken Architektur, Mathematik, Physik, Chemie, Informatik und Wirtschaftswissenschaften zu nennen. Bestanden Ende der 1990er Jahren daneben rund 140 weitere Instituts- und Lehrstuhlbibliotheken ${ }^{3}$, so waren es zur Fusion der Universität mit dem Forschungszentrum Karlsruhe noch rund 80.

Ein wichtiger Faktor bei der Reduzierung war sicherlich der 2006 eingeweihte Neubau der 24-Stunden-Bibliothek. Hier wurden acht größere Bibliotheken der Fakultät Geistes- und Sozialwissenschaften räumlich zusammengeführt. Argumente dafür waren die effizientere Organisation und der deutlich erweiterte Nutzungskomfort rund um die Uhr. ${ }^{4}$ Daneben war und ist die Digitalisierung der wissenschaftlichen Informationen und Publikationen vor allem im Bereich der Forschung der Hauptkatalysator des Wandels hin zu einer stärkeren räumlichen und organisatorischen Integration bzw. Auflösung dezentraler Bibliotheken. In Karlsruhe gelang es - vielleicht schneller als an anderen Standorten - seit Beginn des Internetzeitalters, die Vorteile einer zentralen Verwaltung und Finanzierung elektronischer Datenbanken, Zeitschriften und Monographien deutlich zu machen. Dies führte in den vergangenen 20 Jahren zu einer Konzentration der Personal- und Sachmittel zunächst bei

2 Vgl. den Beitrag von Wilfried Sühl-Strohmenger in diesem Band.

3110 davon mit Beständen unter 5000 Bänden.

4 Kristen, Herbert [u. a.]: Offen ohne Ende: Die neue 24-Stunden-Bibliothek der Universität Karlsruhe. In: BIT online (2006) H. 4. S. 313-320. http://www.b-i-t-online.de/archiv/200604/nach1.htm (25.09.2013). 
der Universitätsbibliothek und später bei der KIT-Bibliothek. Um dies zu begründen, stand und steht immer die messbare hohe Servicequalität und Effizienz im Bereich Informationsversorgung im Mittelpunkt. ${ }^{5}$

Schwieriger als bei den elektronischen Ressourcen gestaltete sich der Weg zur Einschichtigkeit im Bereich der dezentralen Versorgung mit gedruckten Monographien. Diese spielt in den einzelnen Wissenschaftsfächern historisch und aktuell eine sehr unterschiedliche Rolle. Wie viele Monographien als Forschungs-, Lehrstuhl- bzw. Institutsbibliothek vorhanden sein müssen und wie diese zu verwalten sind, wurde zudem häufig vom Selbstverständnis einzelner Forscherpersönlichkeiten geprägt. Im Zuge der oben beschriebenen räumlichen Konzentration in der 24-Stunden-Bibliothek und unter dem Eindruck der raschen Digitalisierung versuchte man, ein System der zentral organisierten Handapparate zu implementieren. Dieses sah vor, die Monographienbearbeitung und die Finanzierung an den Lehrstühlen durch die Universitätsbibliothek zu übernehmen, wenn im Gegenzug bestehende Institutsbibliotheken aufgelöst würden. Um dieses System finanziell nicht vollkommen unberechenbar zu gestalten, wurde eine Obergrenze von 100 Bänden für einen solchen Handapparat festgelegt. In der Praxis zeigte sich, dass trotz dieses Anreizsystems zahlreiche Lehrstühle nicht bereit waren, auf ihre dezentrale Literaturautonomie zu verzichten. Trotzdem wurden vor allem im Zuge von Neuberufungen zahlreiche konstruktive Gespräche geführt und bis zur Fusion mit dem Forschungszentrum 118 Handapparate realisiert.

Bibliothekssystem des Forschungszentrums Karlsruhe

Das Forschungszentrum wurde 1956 durch den Bundesminister für Atomfragen als „Kernreaktor Bau- und Betriebsgesellschaft" gegründet.

$5 \mathrm{Vgl}$. die sehr guten Ergebnisse in der BIX-Dimension Effizienz zusammenfassend dargestellt in: 24 Stunden sind eine Bibliothek. Lernen. Forschen. Kooperieren. Die innovative Bibliothek. Hrsg. von Frank Scholze. 2., überarb. Aufl. Karlsruhe: KIT Scientific Publishing 2013. S. 130. http://dx.doi.org/10.5445/KSP/1000034272 (25.09.2013). 
Bereits bei der Gründung wurde eine "Literaturabteilung" geschaffen, um Funktionen einer Fachbibliothek und eines Dokumentationszentrums zu übernehmen. Zwar wurden auch im Forschungszentrum dezentrale Bibliotheken (Abteilungsbüchereien) vorgesehen, Personal und Organisation waren jedoch von Anfang an zentralisiert. Dies bedeutete, dass Medienerwerbung und Medienbearbeitung grundsätzlich von der Zentralbibliothek durchgeführt wurden - eine funktionale Einschichtigkeit avant la lettre, die an Universitäten erst im Zuge der Neugründungen der 1960er und 1970er Jahre realisiert wurde (z. B. Bielefeld, Konstanz). Bezeichnenderweise wurde für die Bibliothek des Forschungszentrums im Aufbauvermerk von 1957 das Leitbild einer „technischen Werkbücherei eines Industrieunternehmens" formuliert. Es war daher selbstverständlich, dass Servicestrukturen und Dienste zentral bereitgestellt wurden, wobei diese jedoch fortlaufend an die Wünsche der Wissenschaftler angepasst wurden. Dieses System, bei dem die Erwerbungsmittel weitgehend, jedoch nicht vollständig, zentralisiert waren, wurde mit geringen Modifikationen bis zur Fusion des Forschungszentrums mit der Universität beibehalten. Aufgrund der engen Serviceverflechtungen entwickelten nur wenige dezentrale Bibliotheken überhaupt nennenswerte Bestandsgrößen.

Bibliothekssystem des KIT

Die Bibliotheken des Universitäts- und des Großforschungsbereichs stellten bereits zu einem frühen Zeitpunkt des Fusionsprozesses zum KIT konkrete Überlegungen hinsichtlich gemeinsamer Dienstleistungen und einer gemeinsamen Organisationsstruktur an. Schwerpunkte waren hierbei die Schaffung eines gemeinsamen Lizenzraumes für elektronische Ressourcen und Koordination der Zeitschriftenerwerbung.

Für die beiden „Wurzeln” der KIT-Bibliothek wurde deutlich, dass der ehemalige Universitätsteil sein Selbstverständnis aus einem hohen technischen Automatisierungs- und Innovationsgrad bezog (u. a. als erste 24-Stunden-Bibliothek mit vollautomatischer Ausleihe und Rückgabeverbuchung in Deutschland). Gleichzeitig prägten die 
Versorgung der Studierenden und damit der effiziente Massenbetrieb die Arbeitsvorstellung.

Der Großforschungsbereich stellte als Forschungs- und Spezialbibliothek die individuelle Versorgung der Wissenschaftler in den Mittelpunkt. Zudem verstand man sich als kleine hochflexible Dienstleistungseinrichtung mit flachen Hierarchien, die trotzdem eng in ein größeres Ganzes der allgemeinen Forschungsverwaltung eingebettet ist. Im Gegensatz dazu war der Aspekt einer Zentralverwaltung im Universitätsbereich schwächer ausgeprägt.

Erst nach der Entwicklung einer gemeinsamen Leitstrategie und eines gemeinsamen Dienstleistungsverständnisses wurde eine neue, campusübergreifende Organisationsstruktur verwirklicht, die von breiter Akzeptanz getragen war. ${ }^{6}$

Wichtig für die gegenseitige Wahrnehmung als Voraussetzung zur Schaffung eines neuen gemeinsamen Selbstverständnisses und einer neuen Struktur war das Benennen und Akzeptieren unterschiedlicher Rahmenbedingungen, die nicht oder nicht unmittelbar von der Bibliothek beeinflusst werden können. Hierzu zählen u. a. die finanzielle und räumliche Ausstattung, Dienstvereinbarungen und Tarifverträge, unterschiedliche externe Nutzer und andere Einrichtungen der Informationsversorgung. Möglichkeiten der Gestaltung hingegen eröffneten sich bei Zugangsmöglichkeiten und Dienstleistungsangebot sowie internen Prozessen und Policies an beiden Standorten. Hierauf wurde im weiteren Verlauf das Hauptaugenmerk gerichtet.

Das Gruppieren von Diensten und Bereichen wurde in diesem Zusammenhang mehrheitlich als sinnvolle Konsequenz gesehen, um das operative Geschäft bestmöglich zu unterstützen. Die gruppierten

6 Scholze, Frank u. Maja Bailer: Aus dem Schützengraben nach Schätzen graben und nach den Sternen greifen - Der Fusions- und Entwicklungsprozess der KIT-Bibliothek. In: Like It Lead It Change It: Führung im Veränderungsprozess. Hrsg. von Daniela Eberhardt. Heidelberg: Springer 2012. S. 75-84. http://dx.doi.org/10.1007/978-3-642-25623-3_2 (24.09.2013). 
Bereiche wurden schließlich in vier Abteilungen strukturiert: Medienbearbeitung, Benutzung, Publikations- und Mediendienste und Forschungsdienste. In dieser neuen Struktur wurden alle Zuständigkeiten für dezentrale Bibliotheken und Handapparate innerhalb der Abteilung Benutzung der KIT-Bibliothek zusammengeführt.

Erleichtert wurde die Zusammenführung sicher auch dadurch, dass im Großforschungsbereich aufgrund der starken Zentralisierung kein Bibliothekspersonal außerhalb der Hauptbibliothek eingesetzt wurde. So konnte sich ein vergleichsweise kleiner campusübergreifender Bereich als Serviceschnittstelle für alle Belange der dezentralen Bibliotheken etablieren, der entsprechend mit den Fachreferaten und der Medienbearbeitung verzahnt ist. Die in den Fakultäts- und Fachbibliotheken des Universitätsteils Beschäftigten sind fachlich und disziplinarisch in die KIT-Bibliothek eingebunden und Teil des neuen Bereiches.

Die einzelnen Disziplinen oder Fächer spielen in diesem Zusammenhang eine (noch) untergeordnete Rolle. Die Programmorientierte Forschung des Helmholtzbereiches und die freie Forschung des Universitätsbereiches wurden erst in Teilen zu einer neuen Art von Schwerpunktforschung angenähert bzw. neu strukturiert (Materialforschung, Mobilität, Energieforschung). Auch in Zukunft werden Programmforschung, freie Forschung und Schwerpunktforschung nebeneinander existieren, ihre konstitutiven Grundelemente, die Lehrstühle und Institute werden jedoch in neuen KIT-Bereichen gegliedert, die dann auch die akademischen Aufgaben der bisherigen Fakultäten mit umfassen. Eine entsprechende Satzung wurde bereits vom Senat des KIT verabschiedet und muss nun noch durch den Aufsichtsrat bestätigt werden.

Auch wenn die neue Forschungs- und Fächerstruktur des KIT sich noch in einem dynamischen Entwicklungsprozess befindet, ist doch die dezentrale Informationsversorgung innerhalb der Benutzung gut aufgestellt. Hier wird ein intensiver Austausch über operative und strategische Fragen der Informationsversorgung zwischen 
Zentralbibliothek und dezentralen Einheiten gepflegt.

Campusübergreifendes Konzept der Informationsversorgung

Für die Literaturversorgung der dezentralen Einrichtungen wurde ein campusübergreifendes Konzept in Anlehnung an das bisherige Modell des Großforschungsbereiches entwickelt, das der gemeinsame Ausschuss von Senat und Präsidium für die Dienste zur Informationsversorgung (A-IVS) verabschiedete. Die Beschaffung und Verwaltung von gedruckten Monographien in Handbibliotheken wird danach zentral von der KITBibliothek durchgeführt, erfolgt jedoch mit dezentraler Finanzierung durch die Institute bzw. Lehrstühle. Mit diesem neuen Konzept der Handbibliotheken ist ein einheitlicher, wirtschaftlicher und effizienter Beschaffungs- und Lieferservice für Forschungs- und Büroliteratur für alle Mitarbeiter des KIT etabliert worden. Wissenschaftler können dabei direkt über ein Webformular die gewünschten Monographien bestellen und bekommen diese an den Arbeitsplatz geliefert. Alle elektronischen Ressourcen (Zeitschriften, Datenbanken, Bücher), wissenschaftliche Printzeitschriften sowie Lehrbücher werden weiterhin zentral von der KITBibliothek beschafft und verwaltet. Hier war, wie oben beschrieben, bereits bei den Vorgängereinrichtungen des KIT eine weitgehende Konvergenz der Modelle vorhanden.

Traditionelle Institutsbibliotheken im Universitätsbereich sind nach diesem Konzept ein Auslaufmodell, ohne dass sie jedoch mit unnötig hohem Aufwand im Einzelfall aufgelöst würden. Digitalisierung, Effizienz und Serviceangebot der KIT-Bibliothek sprechen für sich und führen zu einer durchgängigen und pragmatischen Informationsversorgung, die auf die Bedürfnisse der Forschenden und Studierenden ausgerichtet ist.

In diesem Sinne wurde im September 2013 von Präsidium und Senat des KIT eine neue „Ordnung für die Informationsversorgung und das Bibliothekssystem des Karlsruher Instituts für Technologie (KIT)“ 
verabschiedet. ${ }^{7}$ Diese schreibt die funktionale Einschichtigkeit im Bibliotheksbereich als Teil des Systems der integrierten Informationsversorgung und Informationsverarbeitung mit einer zentralisierten Verwaltung der Personal- und Sachmittel fest. Zur weiteren Entwicklung heißt es in Paragraph 5 explizit: „Die bestehenden dezentralen Fachbibliotheken werden, wo immer dies angebracht ist, organisatorisch und verwaltungstechnisch zu größeren Einheiten zusammengefasst und nach Möglichkeit auch räumlich integriert."

\section{Zusammenfassung und Ausblick}

Während sich das System der kleinen und kleinsten Institutsbibliotheken im Universitätsbereich des KIT organisatorisch und räumlich immer weiter konsolidiert, stellt sich die Frage nach den noch bestehenden größeren Fakultäts- und Bereichsbibliotheken innerhalb dieses Systems. Nach allen bisherigen Erfahrungen und möglichen Prognosen zukünftiger Entwicklungen erscheint es sinnvoll, diese Bereiche zu funktionalen Lernund Kommunikationsräumen weiter zu entwickeln und die gedruckten Bestände dabei weiter zu verdichten und räumlich zu konzentrieren. Derzeit werden konkrete Überlegungen angestellt, dies in einem zweiten Bauabschnitt der 24-Stunden-Bibliothek umzusetzen. Sowohl der Umfang der Konzentration, als auch der Realisierungszeitraum sind derzeit noch nicht endgültig festgelegt, die Konzepte orientieren sich jedoch am aktuellen Stand der Diskussion zukünftiger Bibliotheksbauten. ${ }^{8}$ 